\title{
Technical Note: Suppression of artifacts arising from simultaneous cone-beam imaging and RF transponder tracking in prostate radiotherapy
}

\author{
Gavin Poludniowski, Steve Webb, and Philip M. Evans
}

Joint Department of Physics, Division of Radiotherapy and Imaging, Institute of Cancer Research and Royal Marsden NHS Foundation Trust, Downs Road, Sutton, Surrey, SM2 5PT, UK

Purpose: Artifacts in treatment-room cone-beam reconstructions have been noted at our center when cone-beam acquisition is simultaneous with radio-frequency transponder tracking using the Calypso 4D system (Calypso Medical, Seattle, WA, USA). These artifacts manifest as CT-number modulations and increased CT-noise. We present a method for the suppression of the artifacts.

Methods: We propose a three-stage post-processing technique that can be applied to image volumes previously reconstructed by a cone-beam system. The stages are: (I) segmentation of voxels into air, soft-tissue and bone; (II) application of a 2D spatialfilter in the axial plane to the soft-tissue voxels; (III) normalization to remove streaking along the axial-direction. The algorithm was tested on patient data acquired with Synergy XVI cone-beam CT systems (Elekta, Crawley, UK).

Results: The computational demands of the suggested correction are small, taking less than 15 seconds per cone-beam reconstruction on a desktop PC. For a moderate loss of spatial-resolution, the artifacts are strongly suppressed and low-contrast visibility is improved. 
Conclusion: The correction technique proposed is fast and effective in removing the artifacts caused by simultaneous cone-beam imaging and RF-transponder tracking.

Key words: prostate radiotherapy, cone-beam CT, Radio-Frequency transponders, tracking, artifacts

\section{INTRODUCTION}

Image-guidance and target tracking are important concepts in modern radiotherapy. Treatment-room cone-beam Computed Tomography (CT) is established as a valuable tool for image-guidance. ${ }^{1}$ Target tracking using commercial radiofrequency (RF) transponder tracking systems is now also possible. ${ }^{2}$ At our radiotherapy center a substantial number of prostate-cancer patients are treated with image-guidance using linac-mounted cone-beam CT (CBCT) and simultaneous transponder tracking using the Calypso 4D system (Calypso Medical, Seattle, WA, USA). It was observed that, for these patients, reconstruction artifacts are apparent in the cone-beam scans. The artifact consists of CT-number modulations superimposed on the patient image slices, and an increase in apparent noise, decreasing the visibility of soft-tissues. The linear-accelerators at our center are Elekta linacs (Elekta Ltd, Crawley, UK). The phenomenon has previously been noted by Zhu et al. ${ }^{3}$ for a Varian linear-accelerator (Varian Medical Systems, Paolo Alto, CA, USA). Such artifacts therefore appear to be a cross-vendor problem. In this Note the artifact is characterized and its almost complete suppression with image-processing is demonstrated. We are aware of only one previous mention of an attempt to correct this technical issue in the research literature ${ }^{4}$ and no published journal articles.

\section{BACKGROUND}


Important components of Calypso tracking system are implanted RF transponders (typically three) and a mobile panel that is positioned above the patient. These features are illustrated in Fig. 1. Two planar projection-images from a conebeam acquisition during Calypso tracking are shown in Fig. 2(a)-(b). These images illustrate that the presence of the Calypso panel in the x-ray beam results in a superimposed image, due to the attenuation of wires within the Calypso panel. This occurs for large sections of the cone-beam acquisition arc. Note the x-ray source was located above and below the patient, respectively, in (a) and (b), resulting in a different degree of magnification of the panel. Also note that Fig. 2(b) shows a second type of artifact: vertical streaks not associated with the attenuation of the Calypso panel. The readout of the flat-panel detector is performed column-wise, so, this latter artifact is likely to be associated with detector readout and probably related to RF interference from the Calypso system. The patterns of readout-streaks appeared random from projection-to-projection but least severe when the detector was furthest from the Calypso-panel. In our investigations it was found that both the readout artifact and the superimposed panel image contributed to degradation of the reconstructions, with the readout artifact only present when the Calypso panel was turned on.

Fig. 3(a)-(c) illustrate the typical reconstruction artifacts that result in the CBCT scan volumes. Axial, coronal and sagital slices are shown for the same patient as Fig. 2(a)-(b). Streak artifacts are visible in each slice and this results in degradation of low-contrast visibility and general image-quality. 


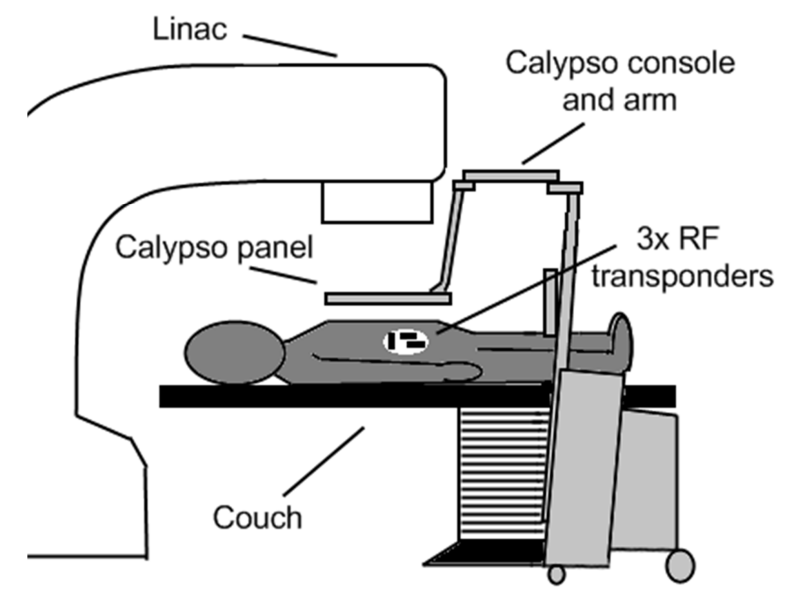

FIG. 1. Illustration of the Calypso 4D tracking system.

(a)

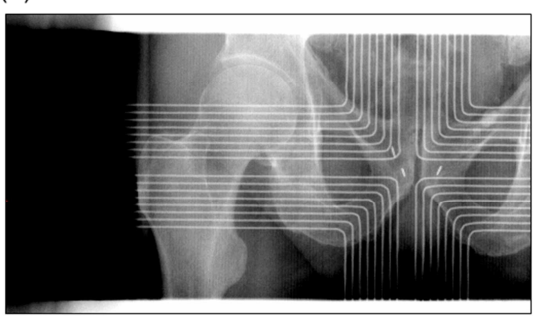

(b)

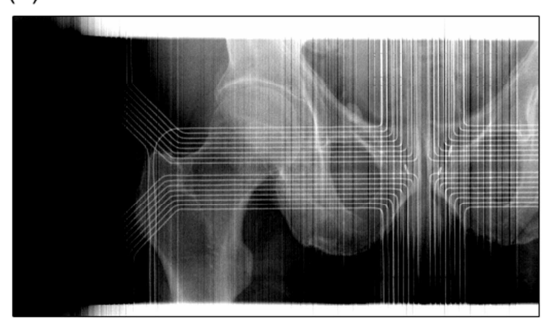

FIG. 2. (a) Planar projection-image acquired with the x-ray source directly above a patient. (b) Planar projection-image acquired with the x-ray source directly below the same patient.

\section{MATERIALS AND METHODS}

A post-processing approach to artifact suppression was adopted i.e. imageprocessing was conducted on the reconstructed image. This directly treats the symptoms of the problem: the unsatisfactory quality of reconstructions. It also largely negates the need to understand the internal workings of the semi-closed system that is a manufacturer's CBCT solution. The processing steps are straight-forward and we are explicit in our description, to allow reproduction by other researchers. 
Patient projections were acquired and reconstructed in the clinic according to the standard medium-resolution protocol used at our center. The flat-panel detector was offset to the medium position with the M10 collimator and bowtie filter inserted. The CBCT volumes were reconstructed on a 410x410x120 matrix $\left(1 \times 1 \times 1 \mathrm{~mm}^{3}\right.$ voxels). As well as using clinically acquired patient images for this study, an acquisition was conducted for a Catphan phantom (The Phantom Laboratory Inc., NY, USA). In the phantom acquisition, the Calypso panel was placed above the phantom, but there were no Calypso transponders present and the panel was not turned on. The same clinical cone-beam scan protocol was used as for the patients. The phantom acquisition was conducted to provide an indication of the affect of the post-processing correction on spatial-resolution.

An original reconstructed image will be denoted, $R\left(x_{i}, y_{j}, z_{k}\right)$, where $x, y$ and $z$ are the right-left, inferior-superior and posterior-anterior directions, respectively. The indices $i, j$ and $k$ denote the voxel number along these three axes. The postprocessing correction consisted of three stages. In Stage I a $k$-means clustering (MacKay 2003) was performed ( $k=3$ ) to segment the voxels into air, soft-tissue and bone. The mean (M) and standard deviation (SD) for soft-tissue were calculated. Two threshold numbers were then defined:

$$
N_{1}=M-3 \cdot S D \text { and } N_{2}=M+3 \cdot S D
$$

A modified image was then created according to the following rule:

$$
R_{I}\left(x_{i}, y_{j}, z_{k}\right)=\min \left(N_{2}, \max \left(N_{1}, R\left(x_{i}, y_{j}, z_{k}\right)\right)\right)
$$

The purpose of Stage I was to reduce large transitions in CT-number e.g. at the air-toskin transition. Sharp "jumps" in CT-number manifest in the high-spatial-frequency 
sector of the frequency domain. It was found that without employing Stage I, sharp jumps in CT-number could lead to edge-transition artifacts after the Stage II filtering. Note that the critical information is retained after Stage I: the soft-tissue voxels-values in which the Calypso artifact is manifest.

The Stage II processing was a suppression of streaks in the axial planes. This was performed in the frequency domain slice by slice. A 2D Butterworth-inspired filter was chosen to differentially-suppress the higher-spatial frequencies:

$$
F(\omega)=\frac{1}{1+\left(\frac{\omega}{\varpi_{c}}\right)^{2 n}}
$$

where $\omega=\sqrt{u^{2}+v^{2}}$ is the magnitude of spatial frequency, $u$ and $v$ are the conjugate variables to $x$ and $y$, and, $\omega_{c}$ is the critical frequency. Note that the maximum spatialfrequency present in the images was, $1 / 2 \tau$, where $\tau=1 \mathrm{~mm}$ is the voxel sampling distance. Inspection of axial slices, for several patients, revealed that the streaking was predominantly oblique to the horizontal and vertical axes. It was therefore considered sub-optimal, in the balance between suppression of artifact and loss of resolution, to use an isotropic filter. Rather, $\omega_{c}$ was made to vary with polar angle, $\phi$, in the 2D Fourier-space. The empirical form,

$$
\omega_{c}(\phi)=\omega_{0}\left(1+\alpha \cos ^{2}(2 \phi)\right)
$$

was chosen where $\omega_{0}$ and $\alpha$ are adjustable parameters, and, where, due to symmetry, $\phi$ may be defined with respect to any axis. In discrete form, the correction-formula for Stage II, is: 


$$
\begin{aligned}
& R_{I I}\left(x_{i}, y_{j}, z_{k}\right) \\
& =\mathrm{FT}_{u, v \rightarrow x, y}^{-1}\left\{F\left(u_{i}, v_{j}\right) \cdot \mathrm{FT}_{x, y \rightarrow u, v}\left\{R_{I}\left(x_{i}, y_{j}, z_{k}\right)\right\}\right\} \text { if } N_{1} \leq R\left(x_{i}, y_{j}, z_{k}\right)<N_{2} \\
& =R\left(x_{i}, y_{j}, z_{k}\right) \text { otherwise }
\end{aligned}
$$

where FT indicates the Fourier transform and appropriate zero-padding is assumed. Note that voxels that were designated as being non-soft-tissue are thus replaced with their original values.

After the Stage II correction, streaks remained in the z-direction, in the coronal and sagital planes. This manifested as "noise" in the axial plane and degraded the image-quality in that plane. The Stage III correction addressed this. This was performed in the spatial domain as follows. First the volume was collapsed in the zdirection by averaging over the $k$-index the CT-number of those voxels determined to be soft-tissue: in this case this was defined to be voxels that satisfied $N_{1} \leq R\left(x_{i}, y_{j}, z_{k}\right)<N_{2}$ plus the additional constraint that they were not adjacent to a voxel that failed that condition. If the collapsed $2 \mathrm{D}$ image is defined as, $I\left(x_{i}, y_{j}\right)$, then a normalization ratio, $r_{\sigma}\left(x_{i}, y_{j}\right)$, was calculated as,

$$
\begin{aligned}
r_{\sigma}\left(x_{i}, y_{j}\right) & =\frac{I\left(x_{i}, y_{j}\right) * * G_{\sigma}\left(x_{i}, y_{j}\right)}{I\left(x_{i}, y_{j}\right)} & & \text { if } I\left(x_{i}, y_{j}\right) \text { is defined } \\
& =1 & & \text { otherwise }
\end{aligned}
$$

where $* *$ denotes a $2 \mathrm{D}$ convolution and $G_{\sigma}\left(x_{i}, y_{j}\right)$ is a Gaussian of width $\sigma$. Note that $I\left(x_{i}, y_{j}\right)$ is undefined if there are no soft-tissue voxels in the line of summation. 140 Then, the final post-processed scan, $R_{I I I}\left(x_{i}, y_{j}, z_{k}\right)$, is given by

$$
R_{I I I}\left(x_{i}, y_{j}, z_{k}\right)=r_{\sigma}\left(x_{i}, y_{j}\right) R_{I I}\left(x_{i}, y_{j}, z_{k}\right)
$$


All post-processing steps were implemented in Fortran 95/2003 and compiled using the Intel Fortran Compiler Version 11.0. The program was run on a desktop computer (3.4 GHz Pentium 4 processor, 2 GB RAM).

\section{RESULTS AND DISCUSSION}

Post-processing parameters that were found to be satisfactory by heuristic optimization were: $\omega_{0}=(150 / 410) / 2 \tau=0.183 \mathrm{~mm}^{-1}, \alpha=0.4, n=8$ and $\sigma=2.0$ mm. Computation time, for a 410x410x120 image, including reading a binary file, applying the three post-processing stages and writing out a corrected binary file, took less than 15 seconds on the desktop PC. Fig. 3(d)-(f) show the same slices as in Fig. 3(a)-(c) after correction using the proposed method. The suppression of artifacts and the improvement in low-contrast visibility is apparent. Prior to and after postprocessing, the mean CT-number can be considered unchanged. The correctiontechnique has been tested successfully on several patients. The necessity of the various stages is demonstrated in Fig. 4(a)-(d), in which another coronal slice for the same patient is illustrated. Fig. 4(a) shows the uncorrected image. Fig. 4(b) shows the image without the Stage II correction applied. The vertical streaks are largely removed but diagonal modulations remains. Fig. 4(c) shows the image without the Stage III correction applied. The diagonal modulations are largely removed but vertical streaks remain. The full correction, shown in Fig. 4(d), suppresses both these artifacts.

Close inspection of the axial slices before and after post-processing (see e.g. Fig. 3(a) and Fig.3(d)) reveals improved low-contrast visibility, combined with a degree of loss in sharpness, as expected. For the Catphan phantom acquisition, the same numbers of line-pairs were discernible in the spatial-resolution module before 
and after processing: three line pairs, or $3 \mathrm{lp} / \mathrm{cm}$. We note the phantom can only provide a coarse measurement at this resolution. However, we can conclude that despite the frequency-domain filtering in Stage II, the high-contrast spatial resolution does not strongly deteriorate. correction of scans that have already been acquired and reconstructed. Naturally, modifications of the equipment or protocols such that the artifacts do not arise in the first place would be preferable. Until that is possible an effective practical solution to the problem has been demonstrated.

(a)

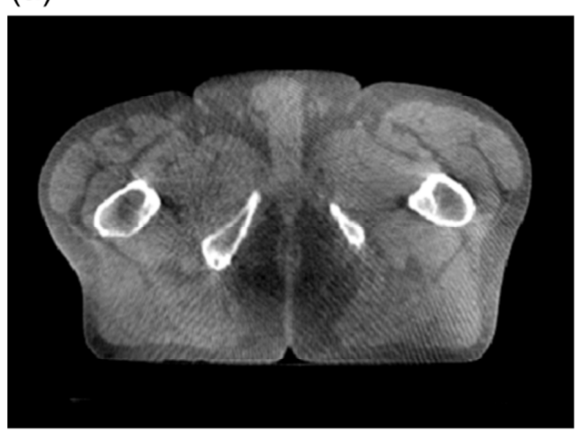

(b)

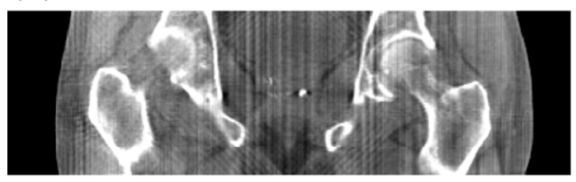

(c)

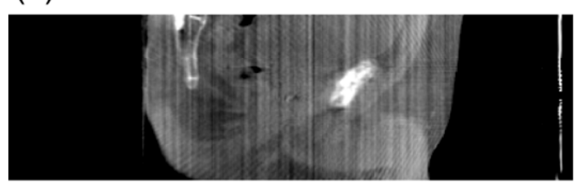

(d)

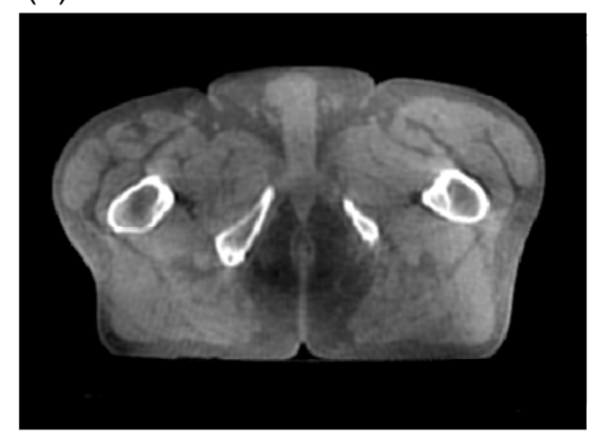

(e)

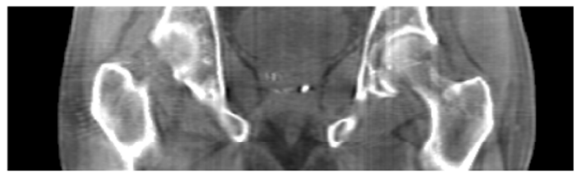

(f)

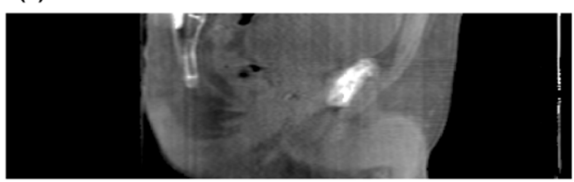

FIG. 3. (a)-(c) Axial, coronal and sagital XVI reconstructions, respectively, of a patient prior to post-processing correction. (d)-(f) Axial, coronal and sagital XVI reconstructions of the same patient after post-processing correction. 
(a)

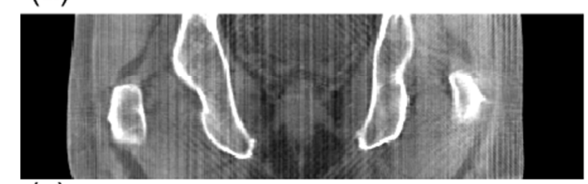

(c)

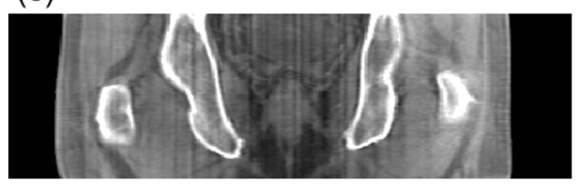

(b)

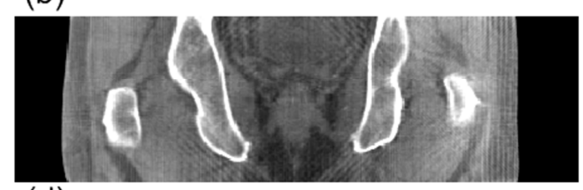

(d)

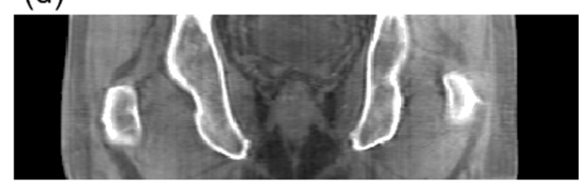

FIG. 4. (a) Coronal slice of a patient prior to post-processing. The same coronal slice after (b) post-processing using only steps I and II, (c) post-processing using only steps I and III and (d) using all three post-processing steps.

\section{CONCLUSION}

Post-processing techniques can almost entirely remove Calypso trackingrelated artifacts in CBCT reconstructions. The computational overhead of such an approach is minimal: a few seconds with a desktop PC.

\section{ACKNOWLEDGEMENTS}

This work was partially supported by research grant C46/A2131 from Cancer Research UK. We thank the Stavros Niarchos Foundation and Calypso Medical, for their support in making this work possible. We also thank Vibeke Hansen for useful discussions and experimental assistance. We thank Jonas Westberg and Carsten Brink of Odense University Hospital, Denmark, for providing code to conveniently decompress XVI .SCAN files. We acknowledge NIHR funding to the NHS Biomedical Research Center.

${ }^{1}$ S. Korreman, C. Rasch, H. McNair, D. Verellen, U. Oelfke, P. Maingon, B. Mijnheer, and V. Khoo, “ The European Society of Therapeutic Radiology and Oncology-European Institute of Radiotherapy (ESTRO-EIR) report on 3D CT-based 
in-room image guidance systems: a practical and technical review and guide," Radiother Oncol 94(2), 129-44 (2010).

${ }^{2}$ T. R. Willoughby, P. A. Kupelian, J. Pouliot, K. Shinohara, M. Aubin, M. Roach III, L. L. Skrumeda, J. M. Balter, D. W. Litzenberg, S. W. Hadley, J. T. Wei, and H. M. Sandler HM, "Target localization and real-time tracking using the Calypso 4D localization system in patients with localized prostate cancer," Int J Radiat Oncol Biol Phys. 65(2), 528-534 (2006).

$205 \quad{ }^{3}$ X. Zhu, J. D. Bourland, Y. Yuan, T. Zhuang, J. O'Daniel, D. Thongphiew, QJ Wu, S. K. Das, S. Yoo, and F. F. Yin, “Tradeoffs of integrating real-time tracking into IGRT for prostate cancer treatment," Phys. Med. Biol. 54(17), N393-401 (2009).

${ }^{4}$ J. Maurer, D. Godfrey, and Q. Wu, "MO-D-BRC-07: Reducing Artifacts in ConeBeam CT Images Caused by the Presence of An Array Used for Tracking Transponders During Radiotherapy," Med. Phys. 38, 3713 (2011).

${ }^{5}$ D. MacKay, "Chapter 20. An Example Inference Task: Clustering” in Information Theory, Inference and Learning Algorithms (Cambridge University Press, Cambridge, 2003). 